\title{
PROBABILITY DISTRIBUTION FUNCTION OF NEAR-WALL TURBULENT VELOCITY FLUCTUATIONS *
}

\author{
ZHOU Ji-fu (周济福)， ZHANG Qiang（张强）， LI Jia-chun (李家春) \\ (Institute of Mechanics, Chinese Academy of Sciences, \\ Beijing 100080 , P. R. China) \\ (Contributed by LI Jia-chun)
}

\begin{abstract}
By large eddy simulation (LES), turbulent databases of channel flows at different Reynolds numbers were established. Then, the probability distribution functions of the streamwise and wall-normal velocity fluctuations were obtained and compared with the corresponding normal distributions. By hypothesis test, the deviation from the normal distribution was analyzed quantitatively. The skewness and flatness factors were also calculated. And the variations of these two factors in the viscous sublayer, buffer layer and log-law layer were discussed. Still illustrated were the relations between the probability distribution functions and the burst events - sweep of high-speed fluids and ejection of low-speed fluids-in the viscous sub-layer, buffer layer and loglaw layer. Finally the variations of the probability distribution functions with Reynolds number were examined.
\end{abstract}

Key words: near-wall turbulence; large eddy simulation; velocity fluctuation; probability distribution function; skewness; flatness

Chinese Library Classification: $0357.5^{+} 2 \quad$ Document code: A

2000 Mathematics Subject Classification : 76F40

\section{Introduction}

Near-wall turbulence plays an important role in environmental engineering due to its great impact on the transport of pollutants, nutrients, sediments, etc. Therefore, people have long been studying the wall-bounded turbulence ${ }^{[1,2]}$, in which the probability distribution function of velocity fluctuations is one of the fundamental issues. Based on the experimental data, Brodkey et al. ${ }^{[3]}$ analyzed the distribution of the turbulent signal in shear flow, showing that there exists skewness and deviation from the normal distribution. Kim et al. ${ }^{[4]}$, Moin \& $\mathrm{Kim}^{[5]}$ simulated channel flows by DNS and LES with Reynolds number (based on the friction velocity and half channel width) of 180 and 640 (i. e., 3300 and

* Received Oct. 18,2004; Revised Jun. 10,2005

Project supported by the National Natural Science Foundation of China ( Nos. 10332050, 10572144 and 10002023) and the Opening Research Foundation of the State Key Laboratory of Water Resources and Hydropower Engineering Science (No. 2003B003)

Corresponding author ZHOU Ji-fu, Associate Professor, Doctor, E-mail:zhoujf@imech.ac.cn 
13800 if based on the centerline velocity and half channel width), respectively. They found that normal distribution is not satisfied. However, the probability distribution of velocity fluctuations was not given in their results, neither the degree of deviation from the normal distribution. Since they only simulated one Reynolds number respectively, the effect of Reynolds number on the probability distribution is not discussed either.

On the other hand, the practical applications require the study of the probability distribution function of velocity fluctuations. For example, there still exists dispute over this issue in the statistical theory of sediment movement ${ }^{[6]}$. In Einstein's bed load theory, the lift force acting on a sediment particle is assumed to follow the normal distribution. However, according to many authors, it is the velocity fluctuation that follows the normal distribution ${ }^{[6]}$. Obviously, because the lift is proportional to the square of the velocity, it is impossible for both to follow the normal distribution. Actually, if the velocity follows the normal distribution, the lift force follows the $\chi^{2}$ distribution.

LES has been recognized as a powerful tool in turbulence research. On the one hand, LES is capable of yielding three-dimensional unsteady instantaneous flow fields to derive any statistical quantities instead of the Reynolds averaged ones. On the other hand, with smallscale motions modeled by sub-grid scale model, LES saves large amount of memory and CPU time comparing with DNS. Therefore, LES method will become a conventional tool in the simulation of complex flows in engineering in the near future ${ }^{[7]}$.

After setting up several databases of turbulent channel flows by LES, we focus the present paper on the characteristics of the probability distribution function of the streamwise and wall-normal velocity fluctuations. We have further calculated the probability distribution function of the streamwise and wall-normal velocity fluctuations. Based on hypothesis test, we have quantitatively evaluated the degree of deviation of the probability distribution function from the normal one. In addition, different characteristics of the probability distribution function in the viscous sub-layer, buffer layer and log-law layer are addressed. And the relations between the probability distribution function and the burst processes, including sweep events of high-speed fluids toward the wall and ejection events of low-speed fluids away from the wall, are illustrated. Finally the effect of Reynolds number on the probability distribution function of velocity fluctuations is shown.

\section{Computational Model and Method}

The geometry in Fig. 1 generalizes the flow condition in natural rivers and channel flows in laboratory. In Fig. 1, $x, y$ and $z$ are the coordinates in streamwise, spanwise and wall-

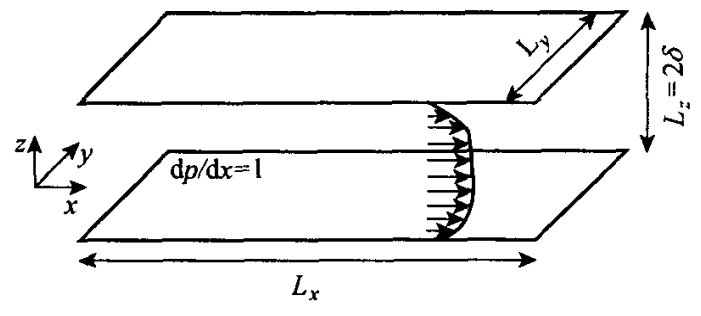

Fig. 1 Sketch of the computational geometry normal directions, respectively. Accordingly, the velocity component in each direction is denoted by $u, v$, and $w$, respectively. $\delta$ is the channel half-width.

In LES, velocity $u$ is divided into large-scale part $\bar{u}$ and small-scale part $u^{\prime}$, i.e. , $u=\bar{u}+u^{\prime}$ and $\bar{u}(\boldsymbol{x}, t)=\int G(\boldsymbol{r}, \boldsymbol{x}) \boldsymbol{u}(\boldsymbol{r}-\boldsymbol{x}, t) \mathrm{d} \boldsymbol{r}$, 
where $\boldsymbol{x}$ is the space vector, $t$ the time, $G(\boldsymbol{r}, \boldsymbol{x})$ the normalized filter function and commonly in the form of $G(r)$, which is independent of $x$.

After filtered and non-dimensionalized by the friction velocity $u_{*}$ and the channel halfwidth $\delta$, Navier-Stokes equations for incompressible fluid become

$$
\begin{aligned}
& \nabla \cdot \bar{u}=0, \\
& \frac{\mathrm{D} \bar{u}}{\mathrm{D} t}=-\nabla \bar{p}+\frac{1}{R e_{\tau}} \nabla^{2} \bar{u}-\nabla \cdot \tau^{r},
\end{aligned}
$$

where $R e_{\tau}=u_{*} \delta / \nu$ is the Reynolds number and $\tau^{r}$ is called the residual stress or sub-grid scale (SGS) Reynolds stress tensor, and its componential form is $\tau_{i j}^{r}=\overline{u_{i} u_{j}}-\bar{u}_{i} \bar{u}_{j}$. In order to close Eqs. (2) and (3), it is necessary to model the contribution of small-scale motion to the large-scale one.

The Smagorinsky ${ }^{[8]}$ model,

$$
\tau_{i j}^{r}-\frac{1}{3} \tau_{k k}^{r}=-2 \nu_{\mathrm{T}} \bar{S}_{i j},
$$

is used here for its simplicity, where

$$
\nu_{\mathrm{T}}=\left(C_{\mathrm{S}} \bar{\Delta}\right)^{2}|\bar{S}|, \quad \bar{S}_{i j}=\frac{1}{2}\left(\frac{\partial \bar{u}_{i}}{\partial x_{j}}+\frac{\partial \bar{u}_{j}}{\partial x_{i}}\right), \quad|\bar{S}|=\left(2 \bar{S}_{i j} \bar{S}_{i j}\right)^{1 / 2},
$$

in which, $\nu_{T}$ is eddy viscosity coefficient, $C_{\mathrm{S}}$ the Smagorinsky coefficient and $\bar{\Delta}$ the filter width. In order to account for the real behavior of the near-wall flow, the Van Driest damping function is used to revise the length scale in the model ${ }^{[9]}$. Hence,

$$
\nu_{\mathrm{T}}=\left(C_{\mathrm{s}} \bar{\Delta}\right)^{2}\left[1-\exp \left(-z^{+} / A^{+}\right)\right]^{2}|\bar{S}|,
$$

where $z^{+}$is the distance from the wall in wall units, $C_{\mathrm{S}}=0.1, A^{+}=25$.

The grids are uniform in the horizontal directions. However, the grids in the wallnormal direction must be stretched to resolve the fine structure in the near-wall region, for example, the burst events. In the lower half of the domain, the grid points locate at

$$
z_{j}=\frac{\mathrm{e}^{\gamma \xi_{j}}-1}{\mathrm{e}^{\gamma}-1}, \quad \xi_{j}=\frac{2 j}{N_{z}} \quad\left(j=1,2, \cdots, N_{z} / 2\right),
$$

where $N_{z}$ is the total number of grid points in the wall-normal direction, $\gamma$ the stretching parameter. The grids in the other half are given by the symmetry about the centerline of the channel. Staggered grid system is adopted in which the points for $u, v$ and $p$ are located midway between those for $w$ given by $\mathrm{Eq}$. (7). The computational parameters are listed in Table $1^{[10]}$.

Table 1 The computational parameters of the present LES

\begin{tabular}{cccc}
\hline$e_{\tau}$ & 180 & 300 & 640 \\
Domain size & $2.5 \pi \delta \times 1.5 \pi \delta \times 2 \delta$ & $2.5 \pi \delta \times 0.75 \pi \delta \times 2 \delta$ & $1.5 \pi \delta \times 0.5 \pi \delta \times 2 \delta$ \\
$N_{s} \times N_{y} \times N_{s}$ & $64 \times 64 \times 64$ & $64 \times 64 \times 64$ & $64 \times 64 \times 64$ \\
$\gamma$ & 2.0 & 2.5 & 4.5 \\
$\Delta x^{+}$(streamwise spacing in wall units) & 22 & 37 & 47 \\
$z_{1}^{+}$(the first grid away from the wall) & 0.9 & 1.0 & 1.0 \\
$\Delta y^{+}$(spanwise spacing in wall units) & 13 & 11 & 15 \\
$\Delta t$ (time step) & 0.001 & 0.001 & 0.0004 \\
\hline
\end{tabular}


Non-slip boundary conditions are used at the walls. The periodic boundary conditions are employed in the horizontal directions. The pseudo-spectral method based on the Fourier transform used for horizontal discretization and the finite difference method for vertical discretization. The Adams-Bashforth scheme is used for the time evolution.

In our previous work, we have shown that the computational results agree well with the DNS of Kim et al. ${ }^{[4]}$. The verification and validation of our method were guaranteed in many aspects, such as the distribution of stresses, turbulent intensities, skewness factors and flatness factor, and grid independence as well (see Ref. [10] for detail). As further work, the current paper focuses on the characteristics of the probability distribution function of the streamwise and wall-normal velocity fluctuations.

\section{Probability Distribution Functions of Velocity Fluctuations}

The characteristics of a random variable are commonly described by the expectation, the variance, the skewness factor $S$ and the flatness factor $F$. The skewness factor $S$ indicates the degree of asymmetry of the probability density function $f$. If $S=0$, the probability density function $f$ is symmetric about the expectation, and the expectation coincides with the mode of the distribution. If $S<0$, the expectation locates on the left of the mode, and the distribution is left-skewed or negative-skewed. Otherwise, if $S>0$, the expectation locates on the right of the mode, and the distribution is right-skewed or positive-skewed. The flatness factor $F$, also called kurtosis, indicates the degree of peakedness of the probability density function $f$. The larger the $F$, the more peaked the distribution about its expectation. Equivalently, the smaller the $F$, the flatter the distribution and the more scattered the distribution of the random variable. For the normal distribution, we have $S=0$ and $F=3$.

In the following sections, we examine the characteristics of the three-dimensional instantaneous velocity field obtained by LES. Because of the symmetry, we only deal with the lower half channel. As is known, wall-bounded flows cover three different regions, namely, the viscous sub-layer $\left(z^{+}<5\right)$, the log-law region $\left(z^{+}>30\right)$ and the buffer layer $\left(5<z^{+}<30\right)$ in between. In different regions, the flow behaves differently. Hence, we naturally focus on the probability distribution functions of the streamwise and wall-normal velocity components, the characteristics of their skewness and flatness in these three regions, and their association with the sweep motions and ejections of the near-wall bursts. Meanwhile, comparison with the normal distribution is also made.

\subsection{Distributions of velocity fluctuations}

It is not difficult to calculate the distributions of the fluctuations from the instantaneous velocity series. Figures 2 and 3 present the probability distribution function $f$ of the streamwise and wall-normal velocity fluctuations at different positions for the case of $R e_{\tau}=$ 180. For comparison, the corresponding normal distributions are indicated by the solid line in the figures. Intuitively, the distributions of the wall-normal components are closer to the normal one than those of the streamwise components. Those in the log-law layer (Fig. 2(a) and Fig. 3(a)) are closer to the normal one than those in the viscous sub-layer (Fig. 2(c) and Fig. 3(c)). In the buffer layer (Fig. 2(b) and Fig. 3(b)), the closeness to the normal distribution of the distributions of both components is between those in the log-law layer and 
viscous sub-layer. The distributions of the wall-normal components are roughly symmetric, and more peaked than the normal distribution in the near-wall region. Obviously, the distribution of the streamwise components is positive-skewed in the viscous sub-layer and negative-skewed in the log-law region.

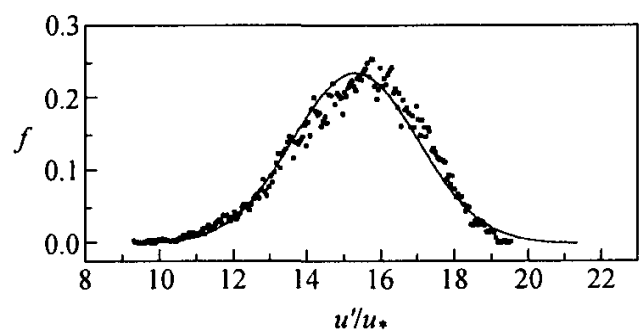

(a) $z^{+}=61$

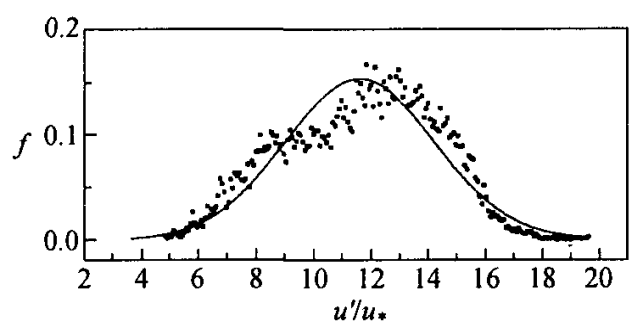

(b) $z^{+}=20$

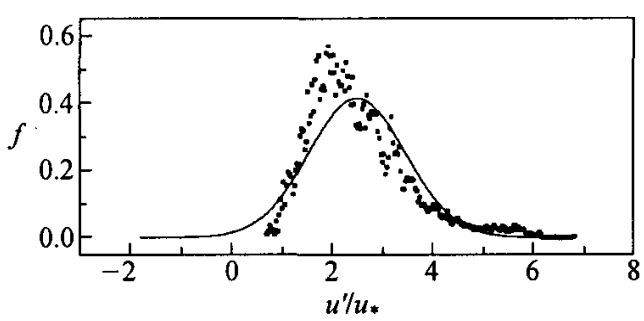

(c) $z^{+}=2.8$

Fig. 2 The probability distributions of the streamwise velocity fluctuations comparing with the normal distribution (Dots: LES results, Solid line: the normal distribution)

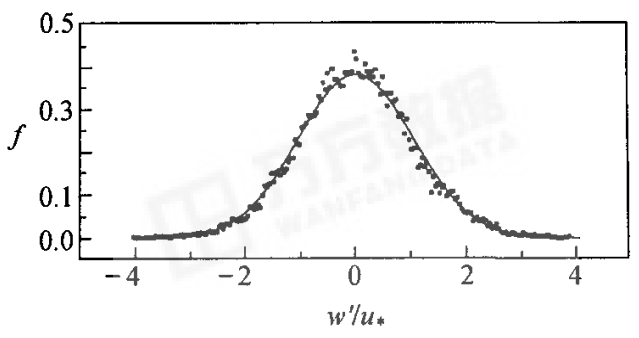

(a) $z^{+}=61$

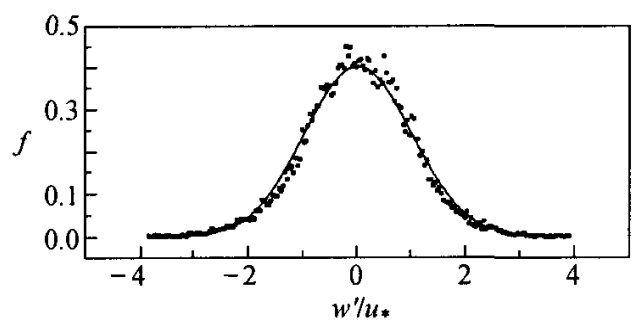

(b) $z^{+}=20$

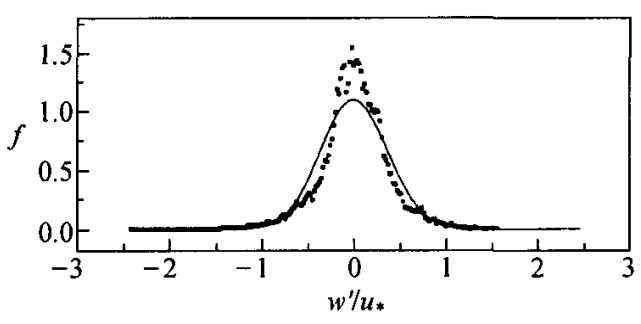

(c) $z^{+}=2.8$

Fig. 3 The probability distributions of the wallnormal velocity fluctuations comparing with the normal distribution ( Dots: LES results, Solid line: the normal distribution )

$\chi^{2}$-test can be used to measure the degree of the coincidence between a distribution and the normal distribution. Given a sample, $\chi^{2}$ can be calculated by the method of segmentfrequency-counting. When $\chi^{2}<\chi_{\alpha}^{2}(k-3)$, the random variable follows the normal distribution (with the mean and variance estimated by the sample) at the significance of $\alpha$, otherwise it does not. The larger $\chi^{2}$ means more deviation from the normal distribution. Here, $k$ denotes the number of the frequency-counting segments. 


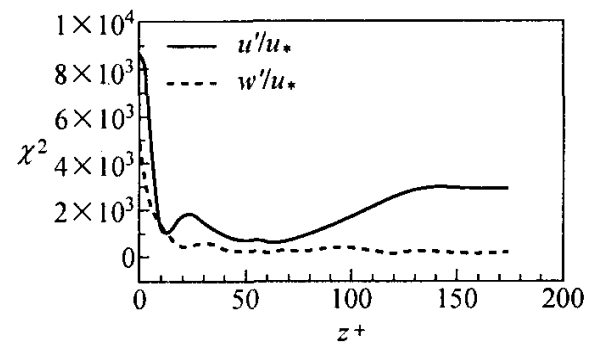

Fig. 4 The variation of the deviation degree of streamwise and wallnormal velocity fluctuations from the normal distribution with the distance away from the wall $\left(z^{+}=180\right.$ locates the center of the channel)

Figure 4 shows the variation of $\chi_{u^{\prime}}^{2}, \chi_{w^{\prime}}^{2}$ with $z^{+}$when $k \approx 20$. Since $\chi_{0.25}^{2}(k-3) \approx 20$, it can be seen in Fig. 4 that $\chi_{u^{\prime}}^{2}, \chi_{w^{\prime}}^{2} \gg \chi_{0.25}^{2}(k-3)$, which means that both the streamwise and wallnormal fluctuations do not follow the normal distribution at a rather high significance level (i.e., at low confidence level). In the viscous sub-layer, the fluctuation distributions deviate greatly from the normal distribution, whereas the deviation is relatively small in the log-law region. By comparison, the distribution of the streamwise fluctuations deviates more from the normal one than that of the wall-normal component.

\subsection{Characteristics of distributions of velocity fluctuations}

The skewness and flatness factors of the streamwise and wall-normal velocity fluctuations at Reynolds number of 180 are shown in Figs. 5 and 6. Obviously, they vary with the vertical position. The DNS data of Kim et al. ${ }^{[4]}$ are also shown in the figures. There is slight discrepancy between the present results of the wall-normal components and the DNS results of Kim et al. ${ }^{[4]}$. This may be attributed to the strongly stretched near-wall grids. In Fig. 5, neither of the skewness factors of the streamwise nor wall-normal fluctuations approaches zero, which indicates that the distributions of both the streamwise and wall-normal fluctuations are not symmetric. The distribution of the streamwise fluctuations skews positively in the region of $z<0.1$ (i.e. , $z^{+}<15$ ), but negatively in the other part of the flow domain. The distribution of the wall-normal fluctuations skews positively in the region of $z<0.05$ (i.e., $z^{+}<10$ ) and $0.2<z<1$ (i.e., $40<z^{+}<$ 180 ), but negatively in the narrow region of $0.05<z<0.2$ (i.e., $10<z^{+}<40$ ). Figure 6 indicates that, as far as the flatness is concerned, the distributions of both components are close to the normal distribution in the log-law layer, yet away from the normal distribution in the viscous sub-layer and buffer layer. However, from both Figs. 5 and 6, neither the streamwise nor the wall-normal fluctuation follows the normal distribution in any regions. This agrees with the result of Brodkey et al. ${ }^{[3]}$.

These non-normal distributions should be attributed to the coherent structures in the near-wall region. In Figs. $7 \mathrm{a}$ and $7 \mathrm{~b}$, the characteristics of such coherent structures are demonstrated by the contours of the streamwise and wall-normal fluctuations in an $x-z$ plane. The high-speed and low-speed regions can be seen in Fig. 7a in the wall region, which corresponds to the downward and upward motions in Fig. $7 \mathrm{~b}$, respectively. These are no other than the sweeps of high-speed fluids and ejections of low-speed fluids in the wall region. Such sweeps and ejections are directional. Therefore, the fluctuations of different components undergo the effect of these events, which leads to the non-normal distributions of the corresponding fluctuations. 


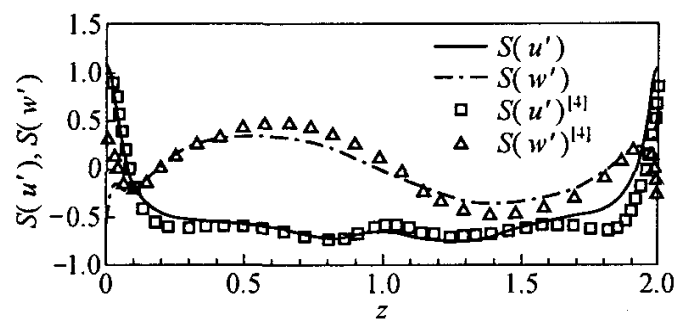

Fig. 5 The variation of the skewness of the streamwise and wall-normal fluctuations with the distance from the wall $\left(R e_{\tau}=180\right)$

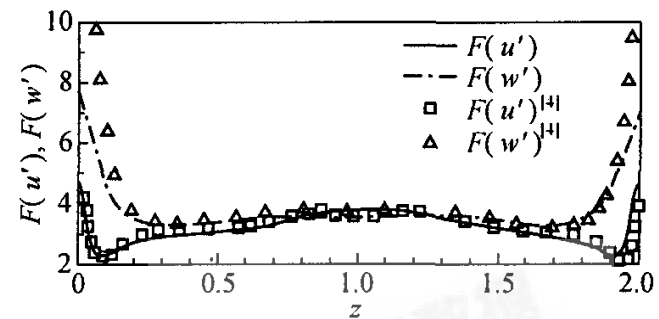

Fig. 6 The variation of the flatness of the streamwise and wall-normal fluctuations with the distance from the wall $\left(R e_{\tau}=180\right)$

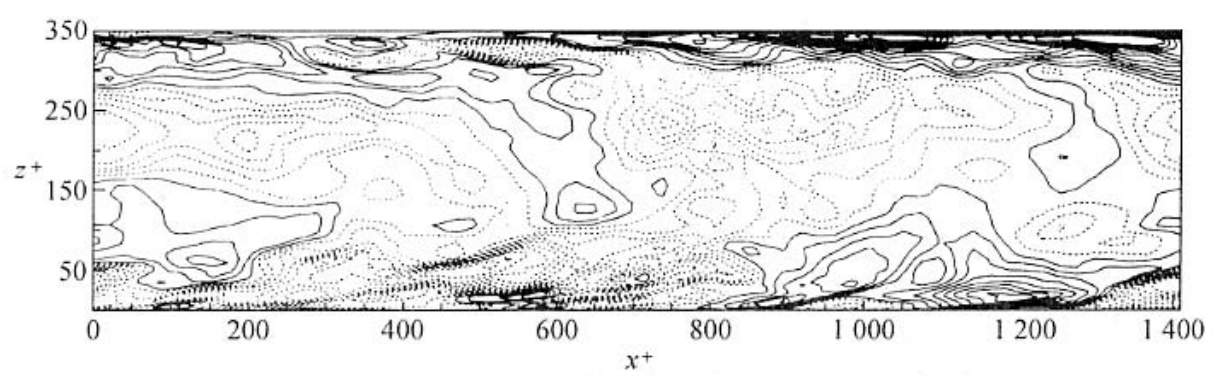

Fig. 7a The contours of the streamwise fluctuations in an $x-z$ plane (solid lines denotes the positive values, standing for the high-speed motions; dashed lines denotes the negative values, standing for the low-speed motions)

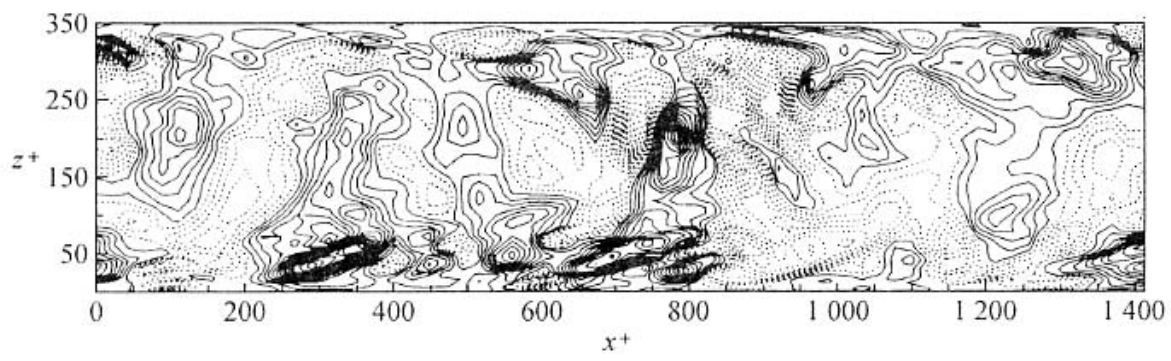

Fig. 7b The contours of the wall-normal fluctuations in an $x-z$ plane (solid lines denotes the positive values, standing for the upward motions; dashed lines denotes the negative values, standing for the downward motions)

The above-mentioned positive-skewed distribution of streamwise fluctuations in the region of $z<0.1$ is mainly due to sweeps of high-speed fluids, whereas the ejections of lowspeed fluids away from the wall lead to the negative skewness of streamwise fluctuations in the outer layer. As for the wall-normal fluctuations, confined by wall, the fluctuations in the region of $z<0.05$ are dominantly upward and the distribution is hence positive-skewed. In 
the region of $0.05<z<0.2$, the effect of sweep events becomes significant, resulting in the negative-skewed distribution. In the region of $0.2<z<1$, the wall-normal fluctuations are mainly controlled by ejection events. Thus, the distribution is positive-skewed. From variations of the flatness factor, we see that the distributions of fluctuations in the wall region are more peaked than the normal distribution, i.e. the fluctuations are mean-valueconcentrated. This is due to the confinement of wall.

\subsection{Effects of Reynolds number}

In order to find out the effect of Reynolds number on distributions of the fluctuations, we have carried out simulation for Reynolds number at 180,300 and 640. The near-wall region decreases in scale (relative to the channel half-width) with the increase of Reynolds number. The grid system is adjusted to sufficiently resolve the near-wall structures, i. e. , to ensure at least three points in the viscous sub-layer with the first one below $z^{+}=1.0$, see Table 1.

Based on the turbulence databases of the flows of these three Reynolds numbers, we compare the wall-normal profiles of the skewness and flatness factors of the streamwise and wall-normal fluctuations of the three cases in Fig. 8. Generally speaking, the tendency of both the skewness and flatness factors vary with the Reynolds number obviously. With the increase of Reynolds number, the skewness factor approaches to zero and the flatness factor to 3 , suggesting that the distributions of fluctuations tend to be the normal distribution. Such effects are more outstanding in the near-wall region. These effects should be attributed to the following two aspects. On the one hand, the larger the Reynolds number, the thinner the viscous sub-layer and the buffer layer, and the wider the log-law region. As a result, in the

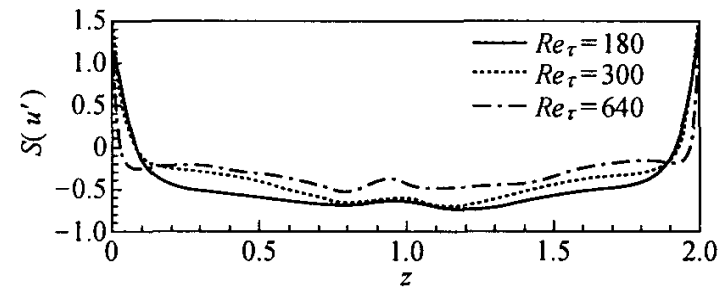

(a)

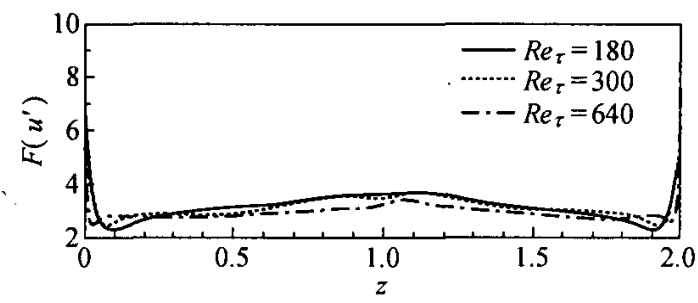

(c)

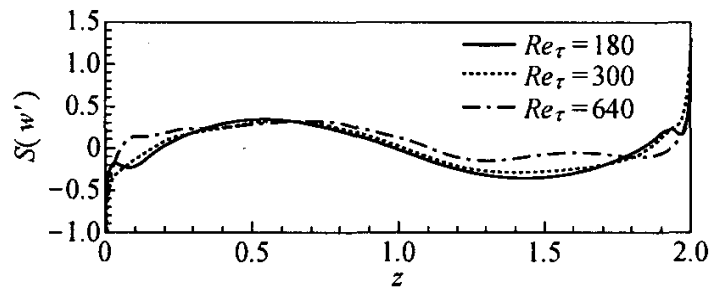

(b)

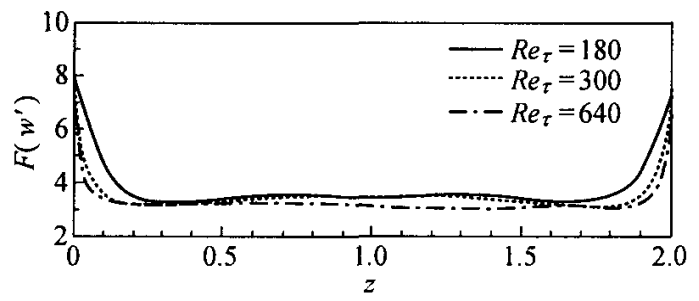

(d)

Fig. 8 The effect of Reynolds number on skewness and flatness of streamwise and wallnormal fluctuations 
near-wall region, part of the viscous sub-layer at the lower Reynolds number turns to be in the buffer layer of the higher Reynolds number. Analogously, the buffer layer at the lower Reynolds number turns to be in the log-law region of the higher Reynolds number. This alteration accounts for the remarkable effects of the Reynolds number on the distributions of velocity fluctuations in the near-wall region. On the other hand, the fluctuations in the loglaw region tend to be more isotropic with the increasing of the Reynolds number. Consequently, the distributions of the velocity fluctuations of the larger Reynolds number are closer to the normal one than those of the lower Reynolds number.

\section{Conclusions}

In the present paper, the large eddy simulations for channel flows are conducted. The probability distribution characteristics of the streamwise and wall-normal velocity fluctuations are analyzed in detail, and compared with the normal distribution quantitatively.

The probability distribution functions of the streamwise and wall-normal fluctuations do not follow the normal distribution. The deviation of them from the normal distribution varies with the distance away from the wall. The larger the distance, the less the deviation.

The distribution characteristics of the streamwise and wall-normal fluctuations are associated with the sweep and ejection events in the near-wall region. The fluctuations in the outer region are predominantly affected by ejections of the low-speed fluids. Thus, the streamwise fluctuation distribution is negative-skewed, whereas the wall-normal fluctuation distribution is positive-skewed. In the near-wall region, the fluctuations are mainly dominated by sweeps of the high-speed fluids, leading to the positive-skewed distribution of streamwise fluctuations and the negative-skewed distribution of wall-normal fluctuations. However, in the very near-wall region, the wall suppresses the downward motions, resulting in a positive-skewed distribution of wall-normal fluctuations there.

The probability distribution functions of velocity fluctuations are obviously affected by Reynolds number, particularly in the near-wall region. The effects of Reynolds number are attributed to the following two aspects. First, with increasing of Reynolds number, part of the viscous sub-layer and buffer layer at lower Reynolds number consecutively turn to be in the buffer layer and the log-law region of the higher Reynolds number, accounting for the remarkable effects of Reynolds number in the near-wall region. Secondly, the fluctuations in the log-law region tend to be more isotropic with the increasing of Reynolds number, thus leading to the distributions closer to a normal one.

\section{References:}

[ 1 ] Sechet P. Guennec B Le. The role of near wall turbulent structures on sediment transport [J]. Wat Res, $1999,33(17): 3646-3656$.

[2] Nino Y,Garcia M H. Experiments on particle-turbulence interactions in the near-wall region of an open channel flow: implications for sediment transport [J]. J Fluid Mech, 1996,326: $285-319$.

[ 3 ] Brodkey R S, Wallace J M, Eckelmann H. Some properties of truncated turbulence signals in 
bounded shear flows[J]. J Fluid Mech,1974,63:209-224.

[ 4 ] Kim J, Moin P, Moser R D. Turbulence statistics in fully developed channel flow at low Reynolds number[J]. J Fluid Mech,1987,177:133 - 166.

[ 5 ] Moin P,Kim J. Numerical investigation of turbulent channel flow [J]. J Fluid Mech,1982, $118: 341-377$.

[ 6 ] Qian Ning, Wan Zhaohui. Mechanics of Sediment Transport [M]. Scientific Press, Beijing, 1986 (in Chinese).

[ 7 ] LI Jiachun. Large eddy simulation of complex turbulent flows : physical aspects and research trends[ J]. Acta Mechanica Sinica,2001,17(4):289-301.

[ 8 ] Smagorinsky J. General circulation experiments with the primitive equations $-I$. the basic experiment [J]. Mon Weather Rev,1963,91:99-164.

[ 9 ] Pope S B. Turbulent Flows[M]. Cambridge University Press, Cambridge, UK, 2000.

[10] Zhang Qiang,Zhou Jifu, Li Jiachun. Burst detection in turbulent channel flows based on large eddy simulation databases[J]. Sci in China, Ser G,2005,35(3):292 - 307 (in Chinese). 